\title{
Design and Implementation of Plant Phenotyping using Smart Electronic Automation
}

\author{
Dileep K. T. \\ Assistant Professor \\ Dept. Of DECS \\ VTU CPGSB, Muddenahalli
}

\author{
Cyril Prasanna Raj P., PhD \\ Research Dean, Dept. of ECE \\ M.S.Engineering College \\ Bangalore, India
}

\author{
Ajey S. S. \\ Assistant Professor \\ Dept of DECS \\ VTU CPGS Kaluburagi
}

\begin{abstract}
The present paper describes the Low-Cost Electronic Automation Unit for Plant Data Acquisition in Plant Phenotyping in which the whole system is made automatic controllable through the sensors. The image capturing of the plant that is the Plant Phenotyping process is done at different angles using a camera and a servo motor. The regular watering system is done automated by obtaining two different results of the water content of the soil present in that particular pot where the plant is present that is through Image Processing, and the Load of the Pot . Automated watering system to the Pot is done through the Solenoid water valve comparing with the results of the water content present in soil.
\end{abstract}

\section{Keywords}

Arduino Mega 2560 ,Servo Motor, DC Motor, IR Sensor, Soil Sensor, Camera, Load sensor.

\section{INTRODUCTION}

In the Smart Electronic Automation Unit for Plant Data Acquisition in Plant Phenotyping shows that the number of plants are placed in individual tray and are mounted on the belt of a DC Motor. To make this system automated, the prototype to take images of the plant at different angles without the presence of human being and to water poured off the plant automatically when the humidity is less than the desired value is developed. In the Electronic Automation Unit for Plant Data Acquisition in Plant Phenotyping shows that in the figure 1 the number of plants are placed in a tray and are mounted on the belt of a DC Motor. The Automation Unit required to sense the presence of plant or the pot requires IR Sensor. For the continuous motion to make all the plants move uniformly, we require DC Motor and for angle rotation of the plant to capture the pictures of the plant at different angles we use Servo Motor. And for capturing of the pictures of the plant we use Camera. Initially, the DC Motor moves the tray of plants on the belt for the process of Plant Phenotyping. The IR Sensor detects the presence of the plant in the system and this is used because there are certain cases where the plant may be present or may not be present in the pot, in this case if the IR Sensor does not detect the plant, the DC Motor will never stop that particular tray at that instant of time and moves forward to the next plant and hence saving the time and power.

The Automation Unit requires an Inductive Proximity Sensor to sense the individual metal tray and an IR Sensor to detect the object that is the Pot. For continuous motion to make all the plants move uniformly, we require a DC Motor and for angle rotation of the Pot to capture the pictures of the plant at different angles we use Servo Motor. Once the DC Motor shaft switches ON, the number of pots placed on that tray starts moving and the IR Sensor and Proximity Sensor gets activated. Once the IR Sensor and Proximity Sensor become high, the DC Motor stops rotating and the Servo motor switches ON. The Pot is rotated at required angles using Servo motor and the image of the plant is captured by the camera and is sent to the receiver either Mobile or PC through Wi-Fi or Bluetooth. At the same time the Load sensor checks for the weight of the Pot and determines the amount of water absorbed by the plant. Later the image of the soil in the pot is taken by the camera to find out the water content in the soil through Image Processing. Once the water content is found to be low compared to the default value, automated watering system is done using Solenoid water valve and this process repeats for the remaining number of pots on the belt.

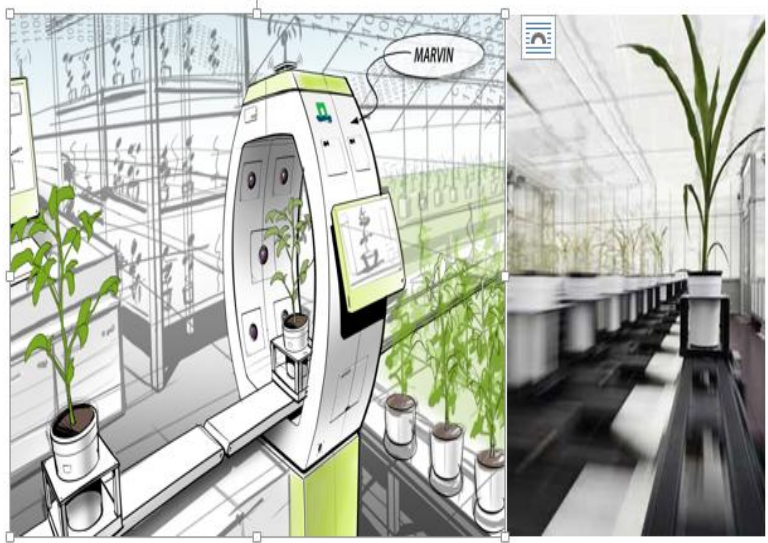

Figure 1: Electronic Automation Unit for Plant Data Acquisition in Plant Phenotyping

\section{PROCEDURE}

Initially the model is set up consisting of a Servo and DC Motors, Conveyor Belt, IR Sensor, Arduino Controller, Camera, Load Sensor, Soil Sensor, Solenoid Water Valve, Wi-Fi or Bluetooth Module.

For the process of Plant Phenotyping, it is required that the images of the plant at different angles have to be taken by the camera and hence we use Servo Motor and is automated at the angles required for the Plant Phenotyping. Camera is used to capture the images of the plant at different angles. Once the pictures are captured from the Camera, the captured image is sent to the cloud using an android application. The images from the cloud can be accessed through Mobile Device at any instant of time. Here, the Electronic Automation Unit for Plant Data Acquisition in Plant Phenotyping method solves the issue of time consumption and replaces the work of the scientist. The captured image signals from camera to cloud and from cloud to the mobile device is to make sure that even if the scientist is not at the lab at that particular instant of time, the scientist ensures that the operation is normal by receiving the signals. The present automation in the industry which is 
going on is economically costlier as it requires around 30 crores for installing in a lab and is time and power consumption and effectively requires man power in the process of capturing the pictures. In this Plant Phenotyping process, the load of the pot is weighed using load sensor and by using certain mathematical calculations the humidity of the soil is found and will be watered through the water solenoid respectively. Once it finds the humidity content of the soil it matches with the default measurements of humidity. If the humidity is less compared to the default readings, the Solenoid Water Valve opens and the particular pot is watered with certain measurements.

\subsection{Model}

In the Smart System Automation Unit for Plant Data Acquisition in Plant Phenotyping shows that the number of plants are placed in individual tray and are mounted on the belt of a DC Motor. The Automation Unit requires an IR Sensor to detect the object that is the Pot. For continuous motion to make all the plants move uniformly, we require a DC Motor and for angle rotation of the Pot to capture the pictures of the plant at different angles we use Servo Motor. Once the DC Motor shaft switches ON, the number of pots placed on that tray starts moving and the IR Sensor gets activated. Once the IR Sensor becomes high, the DC Motor stops rotating and the Servo motor switches ON. The Pot is rotated at required angles using Servo motor and the image of the plant is captured by the camera and is sent to the receiver through the android mobile application. At the same time the Load sensor checks for the weight of the Pot and determines the amount of water absorbed by the plant. Once the water content is found to be low compared to the default value, automated watering system is done using Solenoid water valve and this process repeats for the remaining number of pots on the belt

\subsection{Software Used}

The Software's used for this Design is Arduino 1.6.13, Matlab, and a Camera Application for Mobile Phone or PC. The main coding part has been done using Arduino 1.6.13 and the Image Processing has been done using Matlab and the images of the plant has been transferred to the Mobile or PC through Wi-Fi or Bluetooth through a Camera application.

\section{DESIGN}

The Block Diagram consists of a Transmitter part and the receiver part. The transmitter part consists of two controlling boards that is Arduino Diecimilia or Arduino Mega 2560 and Raspberry-Pi. Arduino board is connected to the components such as DC and Servo motors, IR sensor, Proximity sensor, Load sensor, and Solenoid water valve and Wi-Fi module or Bluetooth module. The Raspberry-Pi board is connected to the camera. The receiver part consists of a mobile phone or a PC to receive the images from the transmitter either through Wi$\mathrm{Fi}$ or Bluetooth. Both the sides that is the transmitter and the receiver together makes a complete block diagram along with the software's used that is Arduino 1.6.13 and Matlab and the self-created camera application. The below figure named as Fig 3 shows the complete picture of the block diagram of the Smart Electronic Automation Unit with all the components. The Block Diagram consists of a Transmitter part and the receiver part. The transmitter part consists of a controlling board that is Arduino Diecimilia or Arduino Mega 2560. Arduino board is connected to the components such as DC motor, Servo motor, IR sensor, Load sensor, and Solenoid water valve. The receiver part consists of a mobile phone or a $\mathrm{PC}$ to receive the images from the cloud. Both the sides that is the transmitter and the receiver together makes a complete block diagram along with the software's used that is Arduino 1.6.13 and an android camera application. The below figure named as Fig 2. Shows the complete picture of the block diagram of the Smart System Automation Unit with all the components.

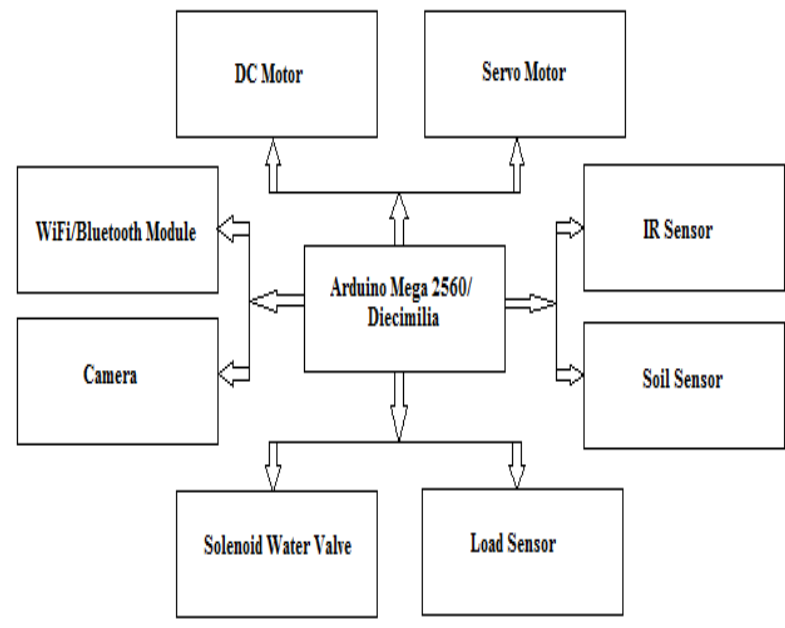

Figure 2: Block Diagram of Smart Automation Unit

\section{FLOWCHART}

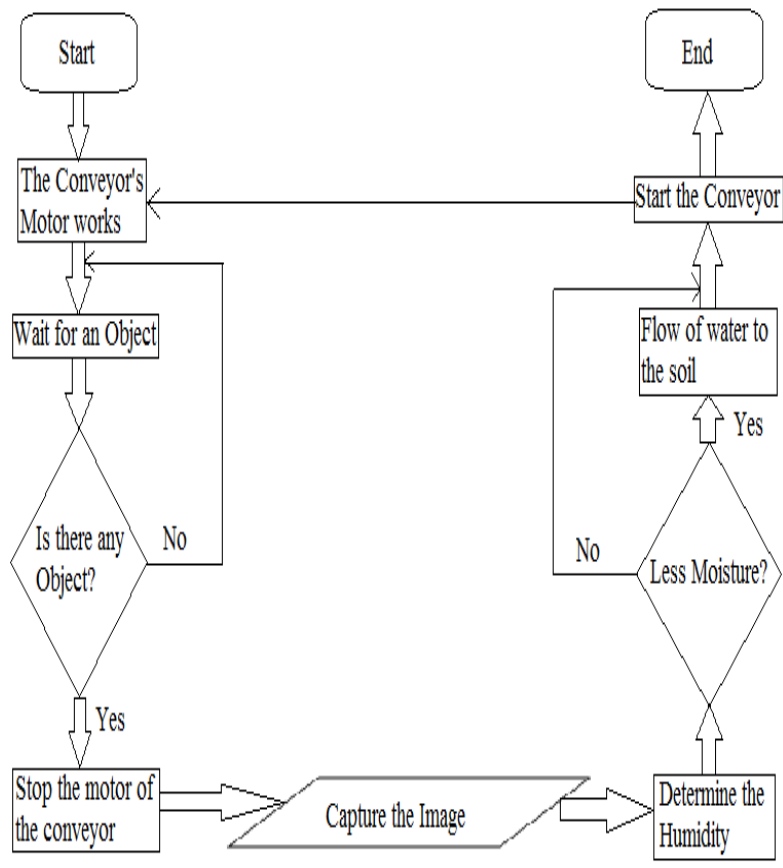

\section{Figure 3: Flow Chart of Smart Automation Unit for Plant} Phenotyping

The project describes the Low-Cost Electronic Automation Unit for Plant Data Acquisition in Plant Phenotyping in which the whole system is made automatic controllable through the sensors. The image capturing of the plant that is the Plant Phenotyping process is done at different angles us in a camera and a servo motor. The regular watering system is done automated by obtaining two different results of the water content of the soil present in that particular pot where the plant is present that is by using soil sensor and the load of the pot . Automated watering system to the pot is done through the solenoid water valve comparing with the results of the 
water content present in soil. The Smart Automated System measures the load of the pot automatically and waters the plant measuring the humidity in the soil and hence replaces the scientist for a major work in less time. The image of the plant is taken by the camera and is image processed to find out the size such as height and width of the plant or stem.

\subsection{Actual Test Setup}

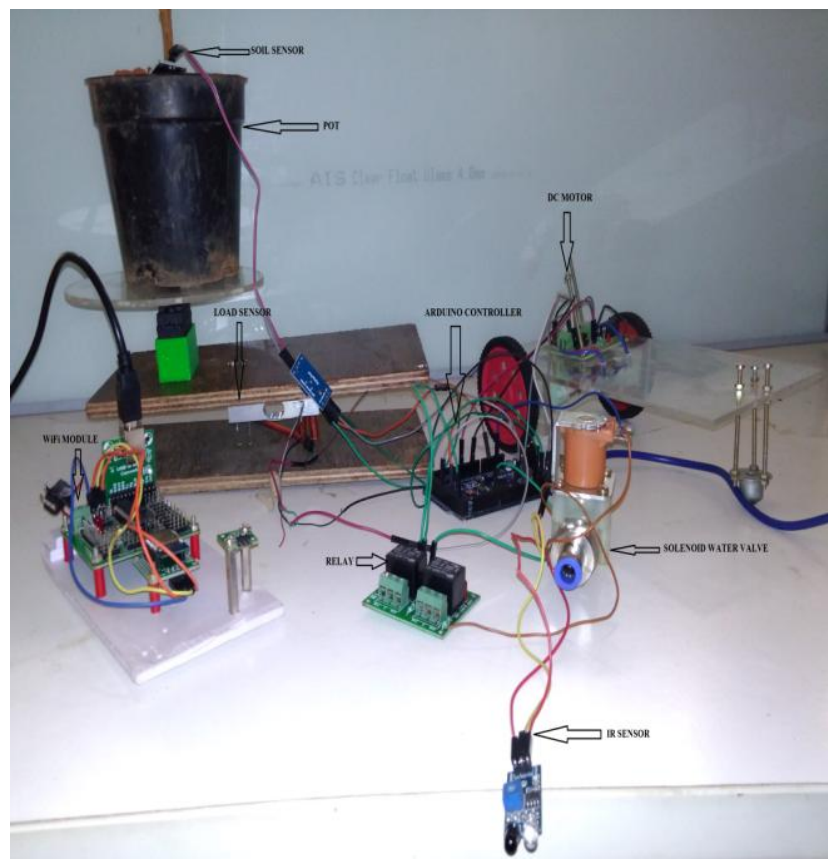

Figure 4: Complete System of Smart Automation Unit for Plant Phenotyping

\section{RESULTS}

The Smart Automated System measures the load of the pot automatically and waters the plant measuring the humidity in the soil and hence replaces the scientist for a major work in less time. The image of the plant is taken by the camera and is image processed to find out the size such as height and width of the plant or stem.

\section{Output Graph:}

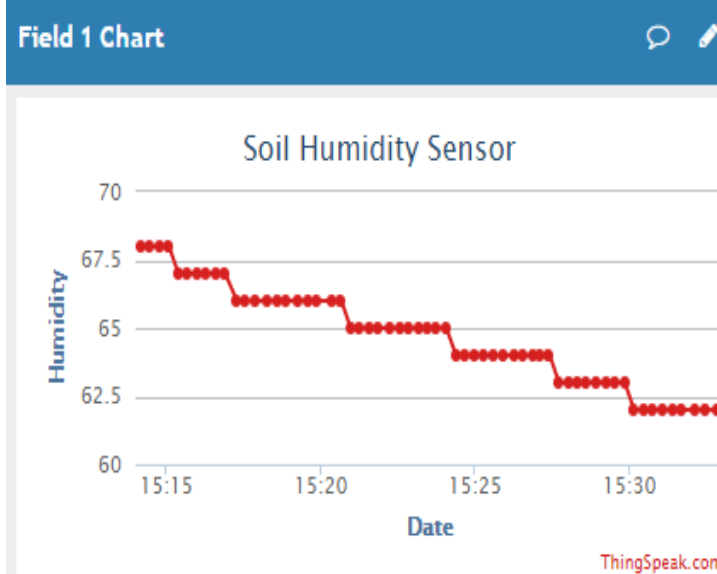

Figure 5: Graph plot of load cell and humidity sensor readings VS Time

$\begin{array}{llll}\text { one reading: } & -0.3 & \text { average: } & -0.3 \\ \text { one reading: } & -0.6 & \text { average: } & -0.6 \\ \text { one reading: } & -0.9 & \text { average: } & -0.8 \\ \text { one reading: } & -1.1 & \text { average: } & -1.1 \\ \text { one reading: } & 1224.5 & \text { average: } & 1225.1 \\ \text { one reading: } & 1225.1 & \text { average: } & 1225.2 \\ \text { one reading: } & 1225.3 & \text { average: } & 1225.3 \\ \text { one reading: } & 1225.3 & \text { average: } & 1225.2 \\ \text { one reading: } & 1225.0 & \text { average: } & 1225.0 \\ \text { one reading: } & 1225.1 & \text { average: } & 1224.9 \\ \text { one reading: } & 1224.9 & \text { average: } & 1224.8 \\ \text { one reading: } & 1224.5 & \text { average: } & 1224.6 \\ \text { one reading: } & 1224.4 & \text { average: } & 1224.5 \\ \text { one reading: } & 1224.3 & \text { average: } & 1260.6 \\ \text { one reading: } & 1400.2 & \text { average: } & 1400.5 \\ \text { one reading: } & 1400.5 & \text { average: } & 1400.3 \\ \text { one reading: } & 1400.2 & \text { average: } & 1400.3 \\ \text { one reading: } & 1400.2 & \text { average: } & 1400.5 \\ \text { one reading: } & 1400.1 & \text { average: } & 1400.1 \\ \text { one reading: } & 1399.8 & \text { average: } & 1399.9 \\ \text { one reading: } & 1399.8 & \text { average: } & 1399.8 \\ \text { one reading: } & 1399.8 & \text { average: } & 1399.8 \\ \text { one reading: } & 1399.9 & \text { average: } & 1400.0\end{array}$

Figure 6: Real time readings of load sensor

\subsection{Equations}

Below equation shows the image processed where the size of the plant such as height and width is found out. $\mathrm{X}$ shows the size in millimeter and $\mathrm{Y}$ shows the value in pixels.

To convert pixels to Millimeter (mm)

$$
\begin{aligned}
& \mathrm{X}=\mathrm{Y}^{*} 0.264583 \\
& \mathrm{X}=\text { value in Millimeter }(\mathrm{mm}) \\
& \mathrm{Y}=\text { value in pixel. }
\end{aligned}
$$

\section{CONCLUSION}

The problems solved by our system is, it is cheaper compared to present system, the plant is watered when the humidity is less than the desired value, the system can be controlled by the scientist without being present in the lab, the pot can be rotated at different angles and the images are transferred through the self-developed camera application either through Bluetooth or $\mathrm{Wi}-\mathrm{Fi}$.

\section{REFERENCES}

[1] C. Granier, L. Aguirrezabal, K. Chenu, S. J.Cookson, M. Dauzat, P. Hamard, J.-J. Thioux, G.Rolland, S. BouchierCombaud, A. Lebaudy, B. Muller,T.Simonneau, and F. Tardieu, "PHENOPSIS, anautomated platform for reproducible phenotyping ofplant responses to soil water deficit in Arabidopsisthaliana permitted the identification of an accessionwith low sensitivity to soil water deficit.," New Phytol.,vol. 169, no. 3, pp. 623-35, Jan. 2006.

[2] M. Bylesjö, V. Segura, R. Y. Soolanayakanahally, A. M. Rae, J. Trygg, P. Gustafsson,S. Jansson, and N. R. Street "LAMINA: a tool for rapid quantification of leaf size and shape parameters.," BMC Plant Biol., vol. 8, no. 1, p. 82, Jan. 2008

[3] A. Hartmann, T. Czauderna, R. Hoffmann, N.Stein, and F. Schreiber, "HTPheno: an image analysis pipeline for high-throughput plant phenotyping.," BMC Bioinformatics, vol. 12, no. 1, p. 148, Jan. 2011.

[4] D. Houle, D. R. Govindaraju, and S. Omholt, "Phenomics: the next challenge.," Nat. Rev. Genet., vol. 11, no. 12, pp. 855-66, Dec. 2010.

[5] R. T. Furbank and M. Tester, "Phenomics--technologies to relieve the phenotyping bottleneck." Trends Plant Sci., vol. 16, no. 12, pp. 635-44, Dec. 2011. 